\title{
Technè
}

La science au service de l'histoire de l'art et de la préservation des biens culturels

46 | 2018

Science et conservation

\section{Restauration et étude comparative de deux dioramas du Suriname (musée des Beaux-Arts de Dunkerque et musée des Cultures guyanaises)}

Restoration and comparative study of two dioramas from Suriname (Musée des Beaux-Arts, Dunkirk, and Musée des Cultures Guyanaises, Cayenne)

Régis Prévot, Laure Cadot, Gérard Collomb, Juliette Langlois, Stéphen Rostain, Claude Steen-Guélen, Yannick Vandenberghe et Ségolène Walle

\section{CpenEdition}

\section{Journals}

Édition électronique

URL : http://journals.openedition.org/techne/419

DOI : $10.4000 /$ techne.419

ISSN : 2534-5168

Éditeur

C2RMF

Édition imprimée

Date de publication : 1 décembre 2018

Pagination : 74-83

ISBN : 978-2-11-152829-1

ISSN : 1254-7867

\section{Référence électronique}

Régis Prévot, Laure Cadot, Gérard Collomb, Juliette Langlois, Stéphen Rostain, Claude Steen-Guélen, Yannick Vandenberghe et Ségolène Walle, "Restauration et étude comparative de deux dioramas du Suriname (musée des Beaux-Arts de Dunkerque et musée des Cultures guyanaises) », Technè [En ligne], 46 | 2018, mis en ligne le 19 décembre 2019, consulté le 24 juillet 2020. URL : http:// journals.openedition.org/techne/419; DOI : https://doi.org/10.4000/techne.419

\section{(c) (†) $\odot$}

La revue Technè. La science au service de l'histoire de l'art et de la préservation des biens culturels est mise à disposition selon les termes de la Licence Creative Commons Attribution - Pas d'Utilisation Commerciale - Pas de Modification 4.0 International. 


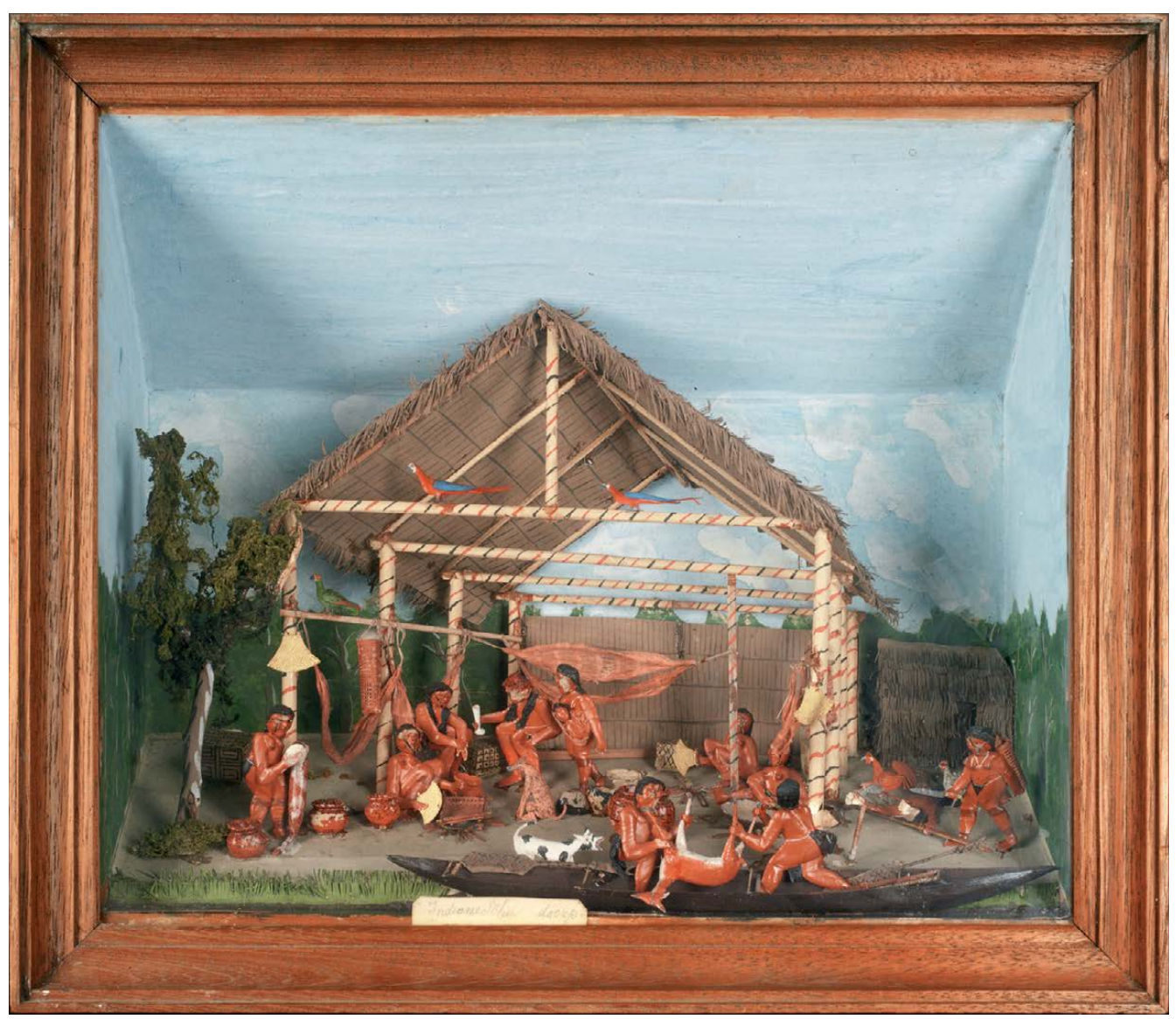

Fig. 1. Vue générale du diorama du musée des Beaux-Arts de Dunkerque avant intervention (inv. BA.1972.00.304). @ C2RMF/M. de Drée.

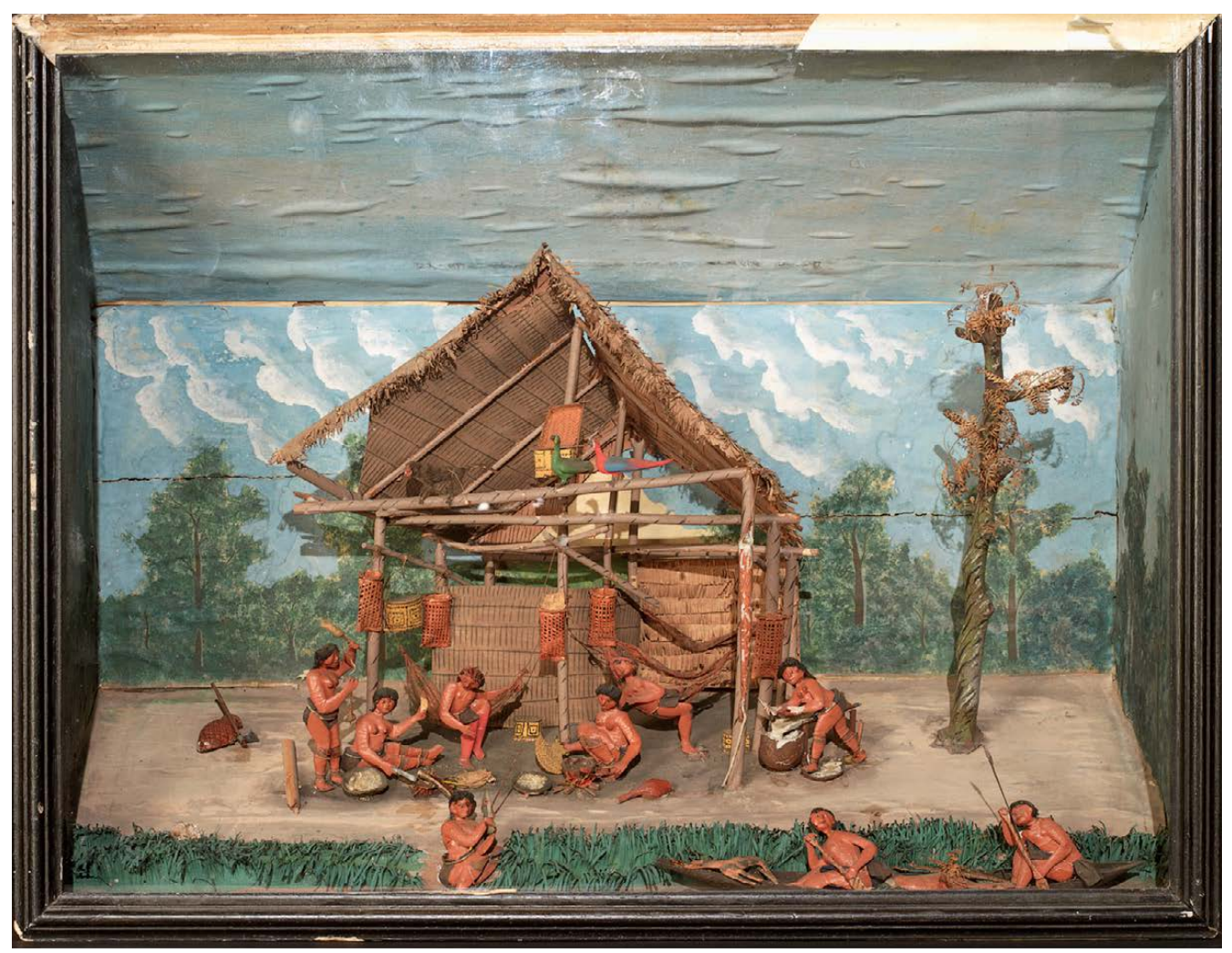

Fig. 2. Vue générale du diorama du musée des Cultures guyanaises avant intervention. (c) C2RMF/G. de Puniet. 
Régis Prévot

Laure Cadot

Gérard Collomb

Juliette Langlois

Stéphen Rostain

Claude Steen-Guélen

Yannick Vandenberghe

Ségolène Walle

\section{Restauration et étude comparative de deux dioramas du Suriname (musée des Beaux-Arts de Dunkerque et musée des Cultures guyanaises)}

Restoration and comparative study of two dioramas from Suriname (Musée des Beaux-Arts, Dunkirk, and Musée des Cultures Guyanaises, Cayenne)

Résumé. Deux dioramas provenant l'un du musée des Cultures guyanaises et l'autre du musée des Beaux-Arts de Dunkerque ont été confiés au C2RMF pour étude et restauration. Une étude comparative a permis de mieux appréhender leur mise en cuvre et de disposer de données techniques de fabrication utiles à la restauration du diorama du musée des Beaux-Arts de Dunkerque. La future restauration d'un diorama du musée des Cultures guyanaises à Cayenne permettra de compléter la collecte des données et, à travers leur confrontation avec les problématiques posées par la restauration du diorama de Dunkerque, de mieux comprendre leurs différences notables d'altération en particulier au niveau des visages. Ces dissemblances sont d'autant plus troublantes que ces illustrations de la vie quotidienne au Suriname se caractérisent plus par leurs similitudes stylistiques, techniques et iconographiques que par leurs différences.

Mots-clés. Diorama, Suriname, Indiens Caraïbes, Kali'na, étude comparative, intervention de restauration.
Abstract. Two dioramas, one from the Musée des Cultures Guyanaises, Cayenne, and the other from the Musée des BeauxArts, Dunkirk, were entrusted to the C2RMF for analysis and restoration. A comparative study provided greater insight into how they were made and technical data about their manufacture that was useful for restoring the diorama in the Musée des Beaux-Arts, Dunkirk. The future restoration of the diorama from the Musée des Cultures Guyanaises will complement the data already collected and, through comparison with the difficulties involved in the restoration of Dunkirk's diorama, will help us to better understand the notable differences in alteration, particularly, in the faces. These dissimilarities are all the more disconcerting since these illustrations of everyday life in Suriname are characterised more by stylistic, technical and iconographic similarities than by their differences.

Keywords. Diorama, Suriname, East Indians of the Caribbeans, Kalina people, comparative study, restoration campaigns.
En mars 2010, la filière Archéologie Ethnologie, du département Restauration du Centre de Recherche et de Restauration des Musées de France (C2RMF), a été sollicitée par le musée des Cultures guyanaises de Cayenne pour accompagner, organiser et suivre la restauration d'un diorama du Suriname ${ }^{1} \mathrm{de}$ la première moitié $d u$ XIX $^{\mathrm{e}}$ siècle, ces opérations étant précédées d'une étude approfondie.

Consécutivement, une demande est parvenue pour un second diorama de même origine et de style similaire appartenant au musée des Beaux-Arts de Dunkerque. Outre le suivi de la restauration, elle concernait aussi la réalisation d'une étude analytique ${ }^{2}$ comparative avec le diorama du musée des Cultures guyanaises.

L'étude a été réalisée sur les deux dioramas alors que seul celui de Dunkerque a pu être actuellement restauré. Tout en relatant les apports de ce premier travail, cet article portera donc essentiellement sur le diorama du musée de Dunkerque. Pour mieux appréhender l'intérêt du sujet traité, un bref historique du diorama, de sa tradition au Suriname et de l'entrée de cet exemplaire dans les collections du musée sera complété par une analyse des scènes et du groupe social représenté.

À l'issue des résultats de l'étude, les complexités techniques liées à la restauration du diorama seront relatées avec les choix et orientations effectués de façon à mener à bien cette opération. À travers les apports de cette étude et de cette restauration, nous souhaitons montrer tout l'intérêt qu'une telle démarche peut avoir pour la connaissance, la compréhension et la préservation de ce type d'objets à ce jour peu étudiés.

Régis Prévot, ingénieur d'études, anciennement au C2RMF (re.prevot@orange.fr). Laure Cadot, restauratrice d'objets ethnographiques (laure_cadot@yahoo.fr). Gérard Collomb, anthropologue, chercheur associé, IIAC-LAIOS (CNRS/EHESS) (collomb@msh-paris.fr). Juliette Langlois, assistante ingénieure, C2RMF (juliette.langlois@culture.gouv.fr). Stéphen Rostain, archéologue, directeur de recherche au CNR (UMR 8096) «Archéologie des Amériques » (stephen.rostain@cnrs.fr). Claude Steen-Guélen, responsable de collections et de la conservation-restauration - musée des Beaux-Arts et LAAC, Dunkerque (claude.steen@ville-dunkerque.fr). Yannick Vandenberghe, technicien de recherche, C2RMF (yannick.vandenberghe@ culture.gouv.fr). Ségolène Walle, restauratrice d'arts graphiques (segolenewalle@yahoo.fr). 


\section{Le diorama : une grande diversité d'illustrations}

Il n'est pas aisé de définir le terme « diorama » qui, dans son sens littéral, signifie «voir à travers ». Le Petit Robert en donne la définition suivante : "Tableau vertical où sont peints des figures, des paysages diversement éclairés. » Des représentations réalisées par Daguerre et le peintre Charles-Marie Bouton à partir de 1822 jusqu'à aujourd'hui, le terme est utilisé pour désigner une grande variété de réalisations. Diversité qui s'exprime par le contenu des sujets traités, des thèmes abordés (vie quotidienne, nature, artisanat, scène militaire, religieuse...), par les variétés de dimensions ${ }^{3}$ de ces illustrations dont certaines peuvent être réalisées à une échelle monumentale, et même par leur nature bidimensionnelle et tridimensionnelle.

Dans la colonie néerlandaise du Suriname, la production de dioramas apparaît dans les années 1790. Elle disparaîtra dans les années 1830 avec l'apparition de la photographie. Il s'agit en fait d'une forme d'illustrations miniaturisées de plantations, de paysages naturels ou urbains (Paramaribo), de scènes de la vie quotidienne des autochtones durant la première moitié $\mathrm{du} \mathrm{XIX}^{\mathrm{e}}$ siècle. Ces représentations en trois dimensions se déploient devant un fond peint en deux dimensions. Elles sont prisées par les « touristes ", les planteurs de l'époque, et se retrouvent aujourd'hui au sein des collections de plusieurs musées néerlandais, suisses et français. Une partie significative de la production est signée et parfois attribuée au botaniste autodidacte hollandais métis Gerrit Schouten $^{4}$ (1779-1834), particulièrement reconnu pour sa maîtrise de cette forme d'expression.

\section{Le diorama de Dunkerque : un témoignage de la culture kali'na au début du XIX ${ }^{\mathrm{e}}$ siècle}

L'intérêt pour l'illustration miniaturisée tire son origine des voyages scientifiques depuis l'époque des Lumières. Le diorama fait partie de ces ensembles d'objets réduits, maquettes ou modèles qui se retrouvent dans bon nombre de cabinets de curiosités du XIX ${ }^{\mathrm{e}}$ siècle avant d'entrer dans les institutions.

Remis au musée en février 1882 par le Dunkerquois Alphonse Bray (1804-1887) ${ }^{5}$ sous l'appellation Indianesche $\operatorname{Dorp}^{6}$, le diorama (fig. 1) décrit plusieurs scènes de la vie quotidienne des Kali'na ${ }^{7}$, groupe d'Indiens caraïbes.

La collection d'objets guyanais remise par les Bray s'avère particulièrement intéressante. On en retrouve certains, miniaturisés, ayant pu appartenir aux Kali'na, tels un éventail à feu woli-woli, et différents modèles de paniers makoki, kulu kulu en vannerie d'arouman ${ }^{8}$. Les dioramas étaient considérés à l'époque de Gerrit Schouten comme des objets de curiosité, rares et à valeur ethnologique. Au-delà du simple témoignage de son aventure guyanaise, on peut supposer que Théodore Bray ${ }^{9}$ ait procédé à une collecte de ces objets dans un souci de visualiser de manière pédagogique la vie quotidienne des caribéens.
Ce diorama montre l'activité d'une famille Kali'na Tilewuyu dans son carbet, lieu de vie et espace du travail domestique. Trois scènes sont juxtaposées, présentant la préparation des galettes de manioc ${ }^{10}$, le retour en pirogue d'une chasse à l'arc, et un moment de détente des hommes de la famille qui consomment vraisemblablement de l'alcool européen, comme le suggèrent le contenant en céramique tournée et l'usage de ce qui semble être un verre. La figuration des différents objets, céramiques, vanneries et la manière de se vêtir sont assez réalistes (si l'on accepte les déformations d'échelle). La décoration polychrome des poteaux du carbet est surprenante et fort peu convaincante, elle ne renvoie à aucune pratique connue chez les Kali'na. S'agirait-il d'une fantaisie d'artiste?

On note la présence d'animaux domestiques, chiens, poules mais aussi aras apprivoisés, et l'on aperçoit un peu à l'écart une petite construction hermétiquement close qui pourrait être l'abri dans lequel s'enferme le chamane lorsqu'il communique avec ses esprits, ou plus simplement un abri complètement clos dans lequel on se protège des moustiques durant la nuit.

Ce diorama présente un témoignage de valeur sur la vie des premiers habitants d'Amazonie à la fin du XIX ${ }^{\mathrm{e}}$ siècle. On est loin des sociétés denses et strictement organisées qui ont occupé les plaines alluviales des rives des grands fleuves et de la côte Atlantique dans le millénaire précédant l'arrivée des Européens. Au moment des premiers contacts, les Kalina Tilewuyu, un peuple de langue caribe, étaient installés sur le littoral des Guyanes. Très affaiblis par les épidémies introduites par les nouveaux arrivants, leur nombre s'est considérablement réduit au cours des XVII ${ }^{\mathrm{e}}$ et XVIII ${ }^{\mathrm{e}}$ siècles, à peine 10 à $15 \%$ des Amérindiens ont survécu.

La scène représentée ici rend compte de cette situation coloniale particulière durant laquelle la vie socio-culturelle des Kali'na a fortement changé, entraînant pour ceux-ci l'adoption de nouvelles stratégies d'acquisition des ressources. En dépit d'une relative naïveté de façonnage des personnages, les détails sont d'une extrême précision. Les maisons et les ustensiles sont d'une fidélité remarquable. Ce sont d'ailleurs les motifs figurés sur les paniers de vannerie et ceux peints sur les poteries qui ont permis l'attribution culturelle du groupe quand la pièce fut extraite des réserves du musée il y a une dizaine d'années. En une seule image figée, l'auteur synthétise quelques-unes des tâches les plus marquantes de la société amérindienne de cette époque. Malgré la présence de rares objets occidentaux, le mobilier représenté est presque entièrement de manufacture amérindienne. Si le troc avec les Européens était déjà depuis longtemps pratiqué, le matériel importé d'Europe n'avait pas encore envahi le monde amérindien. Ces populations vivaient alors un âge transitoire, en partie préservé de l'avidité de notre société. Ce n'est plus le cas aujourd'hui. Cela rend d'autant plus précieuse cette pièce muséale. 


\section{État du diorama du musée de Dunkerque}

Fermé par un châssis vitré, le diorama de Dunkerque (inv. BA.1972.00.304) mesure $42 \mathrm{~cm}$ de largeur sur $35 \mathrm{~cm}$ de hauteur, avec une profondeur de $16 \mathrm{~cm}$. Ses parois intérieures sont recouvertes d'un papier peint illustrant l'environnement naturel de la scène. Les personnages et objets de la vie quotidienne se détachent en trois dimensions sur le fond bidimensionnel.

La caisse du diorama ne présente aucune instabilité structurelle. Le papier peint recouvrant les parois intérieures est poussiéreux. L'encrassement du sol est plus prononcé sous le carbet, devant la petite construction à sénestre et autour de la vannerie à dextre. De nombreuses coulures d'adhésif sont présentes au niveau des éléments structurels du carbet (poteaux, poutres, charpente) tout comme au pied de personnages, d'objets ou sur l'arbre, avec une diffusion dans le papier provoquant des auréoles brunes. La base du feuillage de l'arbre est plus sombre et rigidifiée. Probablement un adhésif qui, en imprégnant le matériau, en a assombri et changé l'état et l'aspect.

La couche picturale du corps des personnages est fissurée et 9 visages sur 11 sont totalement éclatés. Celle-ci, tout comme l'enduit de modelage des visages, est fortement fragmentée et décollée du support cartonné avec des manques de matière importants.

Ces dégradations sont-elles consécutives à la mise en œuvre ou bien traduisent-elles une altération dans le temps du matériau de modelage ou une perte de cohésion entre l'enduit et le support?

Plusieurs terres cuites et vanneries sont désolidarisées ; tout comme un des personnages à sénestre, elles sont mobiles et ne sont plus à leur place. Les débordements d'adhésif brun indiquent que nombre d'éléments ont été refixés sans que
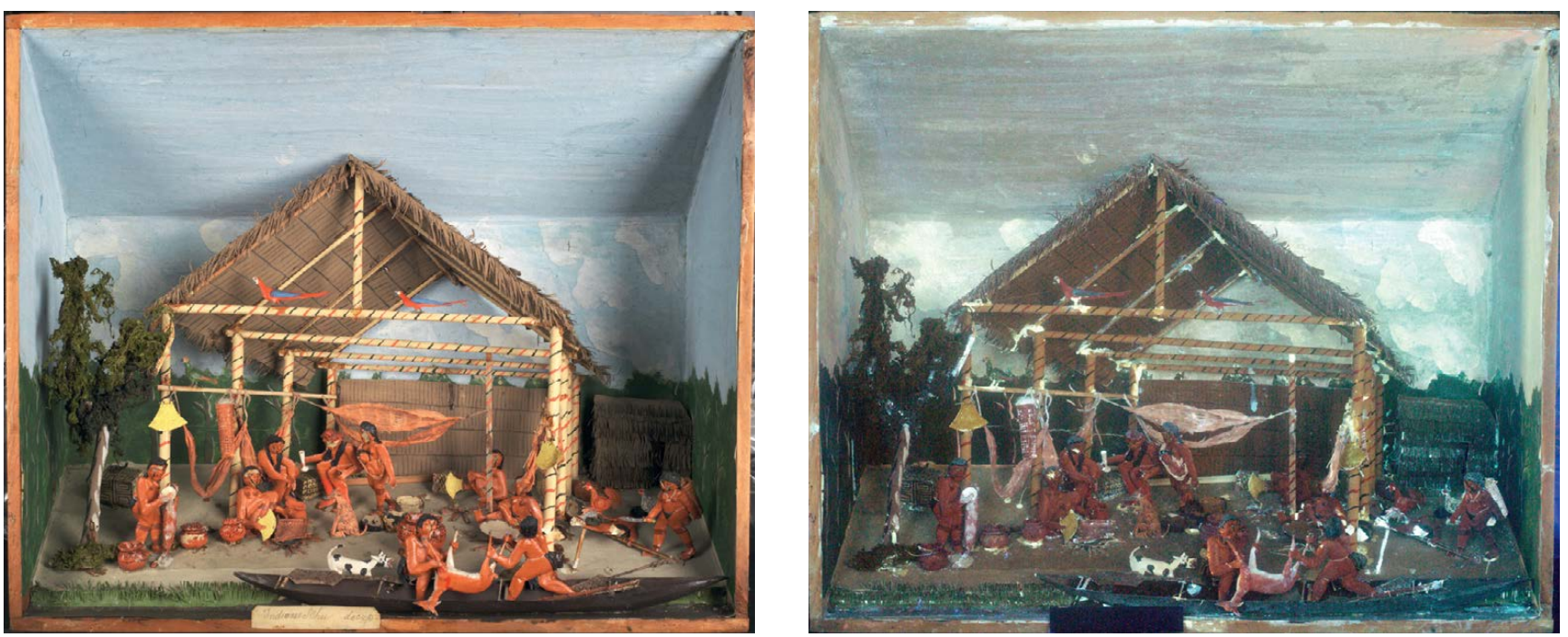

l'on puisse garantir que le positionnement actuel est conforme à celui d'origine.

Nombre de franges du papier représentant l'herbe au premier plan et de fibres en toiture du carbet sont déformées, fragilisées.

\section{État du diorama du musée des Cultures guyanaises}

Acquis en 2010 en salle des ventes, le diorama du musée des Cultures guyanaises (fig. 2) à Cayenne présente des altérations plus nombreuses et plus marquées que celui de Dunkerque. Elles sont majoritairement la conséquence d'un environnement de conservation, en particulier climatique, inadapté (empoussièrement considérable, instabilité hygrométrique avec période durable de forte humidité). Si les mécanismes de dégradation dus à ce type de milieu sont bien connus, ce qui est notable ici, c'est l'état des personnages comparé à ceux du diorama de Dunkerque. Si la polychromie des corps présente un plus grand nombre de fissurations, les visages sont intacts : aucune fissuration, ni soulèvement, ni lacune.

\section{Méthodologie et résultats de l'étude scientifique du diorama de Dunkerque}

Une série d'examens et d'analyses a été pratiquée au C2RMF afin d'obtenir des informations sur la réalisation de ce diorama, tant pour la connaissance de sa mise en ouvre que pour collecter d'éventuelles informations sur l'époque de sa réalisation. Dans le but d'aider à la restauration des visages éclatés des personnages, un intérêt particulier a été apporté à la caractérisation des causes de cette altération.
Fig. 3. Diorama de Dunkerque : détail de la scène en lumière naturelle (à gauche) et sous lumière UV (à droite). () C2RMF/M. de Drée. 


\section{Dossier photographique}

\section{Photographie en lumière directe et en fluorescence sous $U V$}

La première étape a été d'observer le diorama sous lumière naturelle et sous lumière UV. Grâce à la fluorescence des matériaux, cet examen a permis de localiser certaines interventions, comme la présence de colles (d'origine ou de restauration ?). On les visualise au niveau des pattes des oiseaux, de la jonction des montants du carbet ou des objets dans les mains des personnages (fig. 3). L'imagerie sous UV a aussi révélé la présence d'un vernis sur leurs yeux et d'un matériau probablement organique dans leurs cheveux.

\section{Observations sous vidéomicroscope}

Le traitement des phénomènes d'altération des personnages, en particulier des visages, nécessite la compréhension de la mise en œuvre technique. Un des personnages n'étant plus fixé au diorama, il a été possible de l'observer plus en détail, notamment au revers, par microscopie numérique. La figure 4 montre que le modelé des bras, des jambes, des corps ainsi que celui du poisson qu'il tient à la main a été obtenu individuellement par la mise en forme d'une succession de feuilles de papier découpées. Ces éléments ont ensuite été collés entre eux, puis la face visible a été peinte.

Le mode de façonnage des visages est différent de celui des corps. Les visages sont surmodelés sur le support (la succession de feuilles) avant d'être polychromés. C'est probablement le comportement mécanique différent entre la polychromie et le matériau de modelage qui a, dans un environnement instable ou même dès la mise en œuvre, au moment du séchage, engendré ces altérations.

\section{Caractérisation des matériaux inorganiques (de la polychromie et de l'enduit du visage)}

Bien que pour la polychromie, l'analyse par prélèvement soit plus complète, l'analyse par fluorescence $\mathrm{X}$ a été choisie en raison de son aspect non destructif et de la présence d'une polychromie visiblement monocouche. Des pointés d'analyse ont été localisés de façon à être représentatifs de l'ensemble de la palette de couleurs employée par l'artiste et d'approfondir la détermination de la nature des matériaux de la polychromie (fig. 5).

\section{La polychromie}

Après interprétation et comparaison des spectres de microfluorescence $\mathrm{X}$, il a été possible d'établir une liste des pigments employés pour la réalisation de la polychromie.
Le blanc de plomb est observé au niveau des couches blanches (polychromie du chien) et des carnations. Pour ces dernières, il est mélangé avec du vermillon et un pigment au chrome.

Le vermillon est également présent dans les couches picturales rouges, comme pour le plumage des oiseaux.

Le pigment au chrome des carnations est vraisemblablement du jaune de chrome qui est aussi observé pour la couleur jaune des objets suspendus.

La comparaison de plusieurs polychromies vertes (oiseau, herbe et fond peint - fig. 5) permet de retenir le vert anglais, mélange de jaune de chrome et de bleu de Prusse, comme matériau employé par l'artiste.

Le bleu de Prusse est un composé qui n'est pas détecté par fluorescence X, toutefois l'absence de détection d'un autre pigment bleu, ainsi que la visualisation de nodules bleu foncé au vidéomicroscope renforcent l'hypothèse de cette identification. Pour ces mêmes raisons, son utilisation est supposée pour le ciel et les ailes des oiseaux.

\section{Le matériau de modelage des visages des personnages}

L'absence d'accessibilité au matériau constitutif des visages des personnages a nécessité un prélèvement. Réalisé sur le visage du personnage situé sur la gauche de la pirogue et analysé en microscopie électronique à balayage, il a permis de mettre en évidence que la tête des personnages est modelée à l'aide d'une terre ${ }^{11}$ sur une base papier (fig. 4). On observe également la présence de quelques fibres de couleur brune.

\section{Caractérisation des matériaux organiques}

La recherche des matériaux organiques (adhésifs, liants) est réalisée en employant des techniques chromatographiques en phase gazeuse couplées à la spectrométrie de masse (GCMS).

\section{Nature des adhésifs}

Les taches brunes très débordantes correspondent certainement à des refixages. Visibles à l'œil, elles sont confirmées par l'observation sous UV (fig. 3). L'adhésif a été prélevé pour être caractérisé ainsi que celui d'origine, présent sous deux pots et au revers du personnage désolidarisé.

Pour les collages de restauration bruns, tels ceux localisés en bas du pilier et sous le panier noir détaché ou situé au pied du personnage central portant l'enfant, les analyses par chromatographie ont mis en évidence une résine de la famille des Pinaceae sp., caractérisée par la présence de structures abiétanes $^{12}$.

L'analyse menée sur une couche d'adhésif translucide au dos d'un personnage a révélé la présence d'une gomme 
Fig. 4. Détails du revers d'un personnage mettant en évidence le montage des feuilles, l'encollage et l'assemblage des différents membres et l'altération du visage. (c) C2RMF/ Y. Vandenberghe.
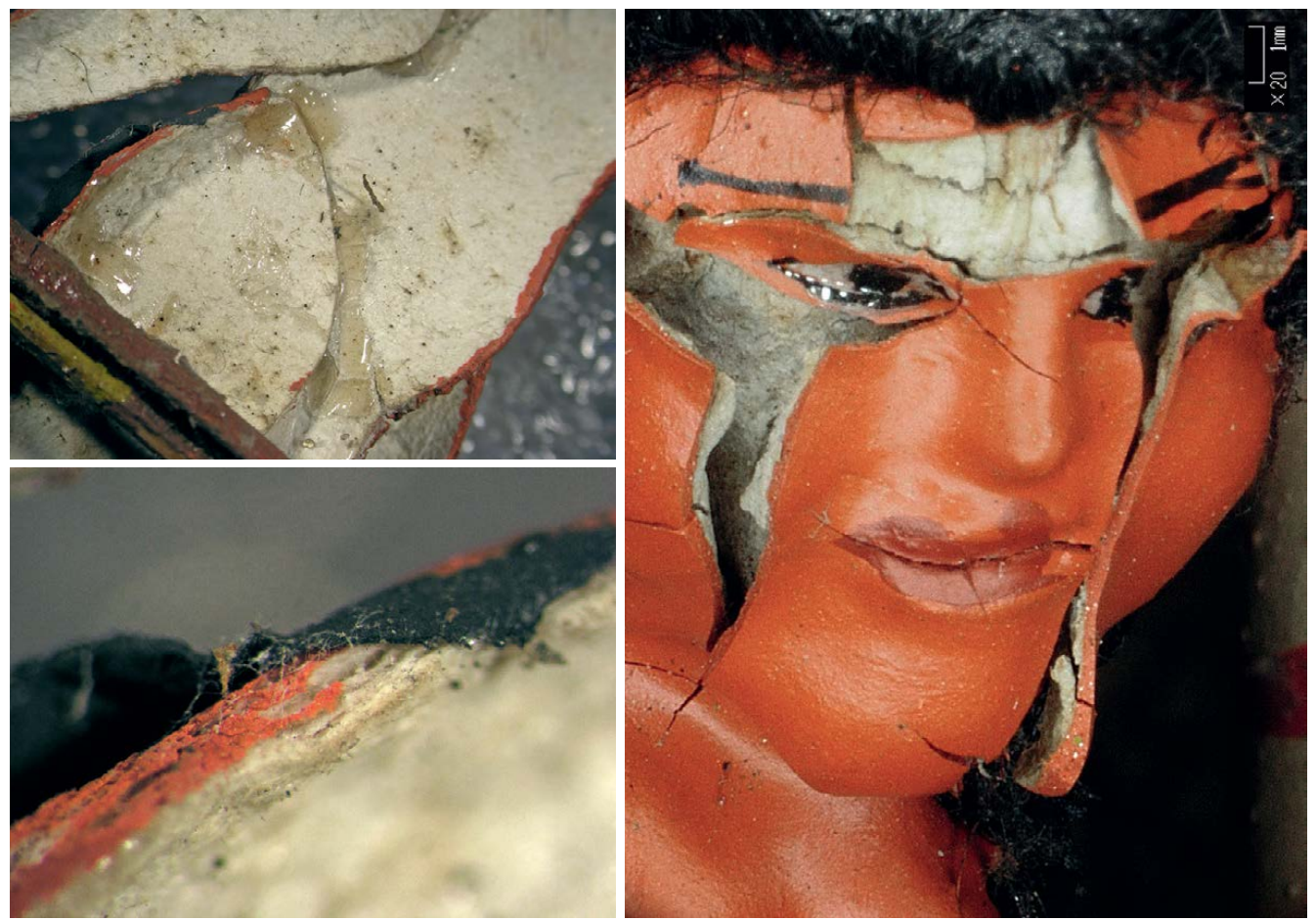

naturelle dont le profil est semblable à celui provenant des espèces Acacias ${ }^{13}$. Il reste cependant difficile de déterminer cette origine botanique avec certitude dans un contexte ethnographique, car il est probable que ce type de profil glucidique soit répandu dans la nature. La mention des espèces Acacias fait ici écho à nos matériaux de référence européens et moyen-orientaux.

\section{Nature des liants}

Le liant de la couche picturale a également pu être caractérisé sur trois éléments distincts : les carnations des personnages, la polychromie d'un panier et un brin d'herbe du décor. Dans les trois cas, les analyses ont révélé la présence de gomme arabique, liant habituellement présent dans les gouaches.

\section{Comparaison des deux dioramas}

Les similitudes entre le diorama de Dunkerque et celui de Cayenne ne se résument pas à des comparaisons de styles. En effet, les analyses mettent en évidence des matériaux et une mise en ouvre similaires. Ainsi, les pigments et la nature des liants et adhésifs sont les mêmes. Seul point de divergence, la mise en ouvre des personnages. S'ils sont également constitués d'un assemblage de fragments de feuilles de papier, les visages, qui ont conservé leur intégrité, sont peut-être ${ }^{14}$ réalisés différemment. De plus, on peut noter un détail concernant la finition de la polychromie, un vernis, présent sur les yeux des personnages des deux dioramas, est également attesté sur les tétons des personnages du diorama de Cayenne.

\section{Problématique et intervention de restauration sur le diorama de Dunkerque}

Pour intervenir sur ce type d'œuvre, une des principales difficultés a été de trouver une équipe à même de donner une réponse appropriée à la demande en termes de protocole et de méthodologie. Un premier appel d'offre s'est révélé infructueux en raison de l'abandon des candidats ou du fait de protocoles d'intervention plus approprié au diorama muséal de grandes dimensions qu'à une petite illustration intimiste. Une réponse adaptée a pu être obtenue en faisant appel à une restauratrice d'objets ethnographiques, Laure Cadot, qui, à notre demande, s'est associée avec une restauratrice d'arts graphiques, Ségolène Walle.

La configuration spatiale du diorama, relevant à la fois de la 2D, pour les décors de fond peints, et de la 3D, pour les éléments en volume venant s'insérer dans ce décor, a nécessité le recours à des méthodes de traitement mixtes et adaptées à chaque zone. Outre la fragilité des matériaux en présence, les difficultés d'accès et l'impossibilité de démonter en raison de la haute sensibilité du tracé à la gouache au traitement par différents solvants constituaient des contraintes supplémentaires dans l'établissement des protocoles. Le degré d'intervention minimal s'imposait de fait comme la seule option envisageable dans un souci d'homogénéité du résultat final 
autant que dans celui du respect de l'histoire de cet objet complexe.

\section{Nettoyage, retrait des surplus d'adhésifs, remise en forme et consolidation}

Des solutions de nettoyage à sec ont été privilégiées autant que possible sur les papiers peints, notamment en raison de la fragilité du support sec et cassant, de la réactivité de la polychromie et des risques de décollement qui n'auraient pu être repris ensuite qu'à grande peine. Le dépoussiérage a été réalisé au pinceau doux avec micro-aspiration et complété par un léger décrassage à sec à l'aide d'éponges cosmétiques. auréoles les plus prononcées.

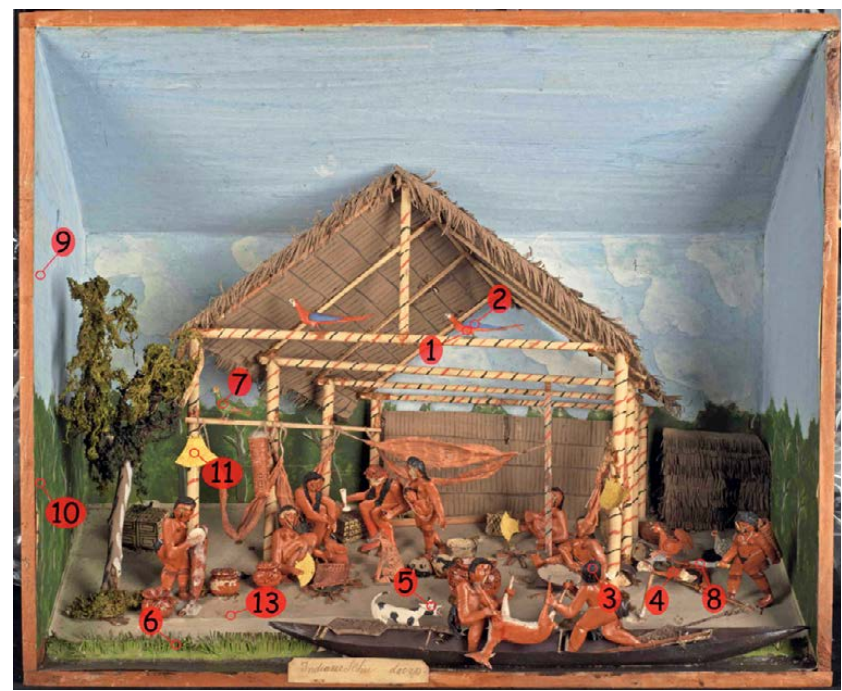

Les nombreux amas d'adhésifs visibles sur le sol, caractérisés comme récents, ont été dégagés mécaniquement au scalpel sous lunettes loupes, après avoir été ramollis à l'acétone. Les amas et coulures sur les montants du carbet ont été atténués avec la même méthode.

L'extérieur de la caisse a pu être gommé à la gomme latex et à l'éponge de carrossier à faible rétention d'humidité. Les résidus de bande kraft ont ainsi pu être dégagés facilement grâce à la légère humidification produite par le passage de l'éponge.

Ce diorama présentait aussi un certain nombre d'éléments déformés, principalement les décors en papier frangé (fibres de la toiture du carbet et herbes sur le devant de la scène). Un léger passage d'un pinceau imbibé d'un mélange eau éthanol (50:50) a permis leur remise en forme par légère pression réalisée à l'aide d'une spatule ou aux doigts.

Les brins fragilisés ont été consolidés par un mélange de colle d'amidon de blé et de méthylcellulose (50:50). Les brins cassés ont été renforcés à l'arrière par un papier japonais très fin $\left(8 \mathrm{~g} / \mathrm{m}^{2}\right.$, papier pure fibre de kozo $)$.

\section{Traitement des visages}

Le choix du traitement des visages des personnages (fig. 6) a donné lieu à un dialogue fructueux entre l'équipe de restaurateurs et les responsables du suivi scientifique de la restauration. L'objectif visait à stabiliser la couche picturale et à retrouver une lisibilité satisfaisante sans dissimuler le caractère technologique de cette dégradation.

Les bords de lacunes et les soulèvements ponctuels ont été légèrement ramollis par nébulisation d'eau déminéralisée puis refixés par imprégnation d'une solution d'Acryl 33

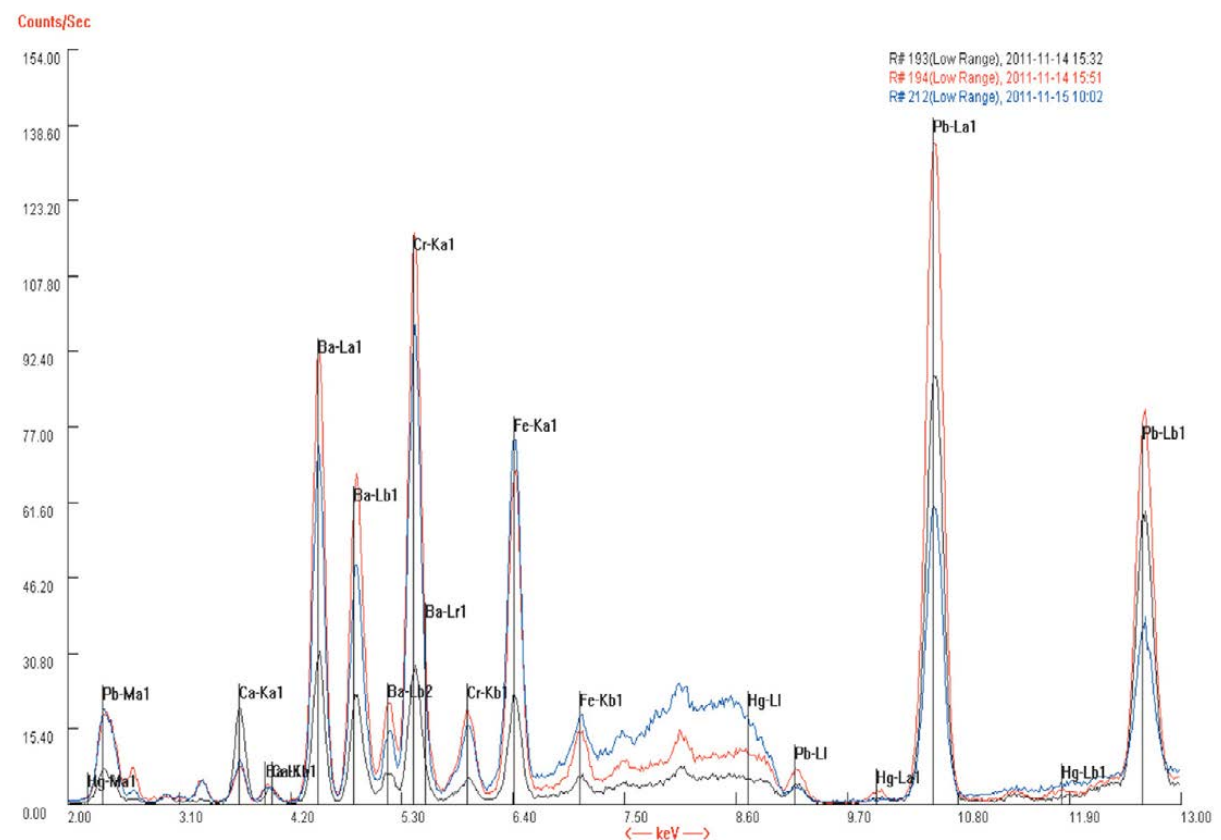

Fig. 5. Photographie de l'œuvre avec la localisation des pointés d'analyses en micro-fluorescence $\mathrm{X}$ et spectres EDS du vert de l'herbe (pointé 6 spectre rouge), du vert de l'oiseau (pointé 7 - spectre bleu) et du vert du fond peint (pointé 10 - spectre noir). (c) C2RMF/M. de Drée/Y. Vandenberghe. 
(résine acrylique en dispersion aqueuse) à $2 \%$ dans un mélange eauéthanol (50:50), traitement choisi à l'issu de tests préalables. L'adhésif sélectionné devait en effet combiner une faible viscosité pour une bonne imprégnation du substrat et un bon pouvoir collant, les possibilités de mise sous poids pendant le séchage étant nulles. Les lacunes laissées après ré-application des soulèvements n'ont volontairement pas été remises à niveau, mais simplement retouchées à l'aquarelle afin d'harmoniser les visages. Ce même protocole a été appliqué avec succès au refixage et à la retouche des lacunes de polychromie sur les céramiques.

\section{Repositionnement des éléments désolidarisés ou mobiles}

Sur les treize éléments désolidarisés avant ou en cours de restauration, onze ont pu être remis en place, les deux qui restaient (une bulle de colle peinte et une traverse en fibre végétale n'appartenant vraisemblablement pas au décor) étant conservés à part. Les trois quarts étaient localisés avec certitude sur la foi d'anciennes traces de fixation ou de logique d'agencement (perroquet sur la traverse du carbet, personnage au poisson au niveau de la languette laissée libre...). En concertation avec l'équipe chargée du suivi scientifique ${ }^{15}$, les éléments restants (une jarre, un faisceau de lance, une bûchette) ont été placés à des emplacements lacunaires et cohérents avec la représentation de la scène en s'appuyant sur l'observation d'autres dioramas similaires. Ils ont été refixés à la Jade $403^{\circledR}$ (copolymère d'éthylène et d'acétate de vinyle (20/80) en émulsion aqueuse) parfois chargée avec des micro-billes de verre afin d'assurer une meilleure stabilité.

Les incohérences, comme le tamis à manioc en vannerie positionné sur un foyer en combustion ou le hamac, situé derrière le personnage assis, ont été laissées en l'état. Aucune trace, aucune information ne permettait de déterminer avec certitude le caractère non original des positionnements. Il n'était de ce fait pas envisageable d'y remédier, en particulier pour le hamac dont les attaches étaient trop courtes.

Afin de garantir sa bonne conservation dans le temps, la vitrine a été équipée d'un verre anti-UV, anti-reflet (verre Schott MirogardMagic Plus ${ }^{\circledR}, 2 \mathrm{~mm}$ ), au moment du remontage de la façade vitrée (fig. 7). Pour éviter que la vitre ne touche les éléments en bordure, comme c'était le cas à l'origine, le cadre a été équipé de rehausses.

\section{Conclusion}

Grâce aux échanges entre l'équipe suivant ce projet, les restaurateurs et des spécialistes (archéologue, anthropologue de l'histoire des cultures d'Amazonie), il a été possible de disposer des informations indispensables à l'interprétation, à la compréhension et à la restauration de ce témoignage en 3D. Elles permettaient, pour les éléments défaits ou mal repositionnés, de mieux en retrouver l'emplacement d'origine, mais aussi de mieux comprendre, dans cet environnement, la présence quelque peu surprenante d'un verre en cristal ou 
celle des repeints des jambes de la femme kali'na ${ }^{16}$ au centre de la composition.

Les réponses apportées par le travail d'analyse et d'étude réalisé par le C2RMF et les échanges qui en ont découlé, tout en apportant des informations plus précises sur les éléments constitutifs et les techniques de mise en œuvre, ont donné aux restauratrices le moyen d'adapter au mieux leur traitement.

Outre la préservation de l'intégrité de l'objet par sa restauration, de façon indéniable, l'objectif commun à tous les intervenants a été de comprendre le message contenu dans ce document en trois dimensions pour, à travers cette illustration, sauvegarder la mémoire de cette culture kali'na et celle de ses coutumes de vie aujourd'hui disparues.

L'étude comparative menée ici, même modeste, a permis d'apporter un éclairage sur certaines techniques de mise en œuvre. Son extension à un corpus plus large et diversifié permettrait, outre la sauvegarde de ces objets de mémoire, d'apporter des réponses plus étendues sur les techniques et les traditions artistiques de cette culture à cette époque.

\section{Remerciements}

Les pilotes de ce dossier, en l'occurrence Claude SteenGuélen et Régis Prévot, souhaitent exprimer leurs sincères remerciements à Dominique Robcis et Thomas Calligaro pour leur expertise dans l'étude par fluorescence X, à Stéphen Rostain et Gérard Collomb pour leur participation à cet article, ainsi qu'à Margrit Reuss du Volkenkunde de Leiden pour les informations qu'elle nous a généreusement communiquées tout au long de ce travail.

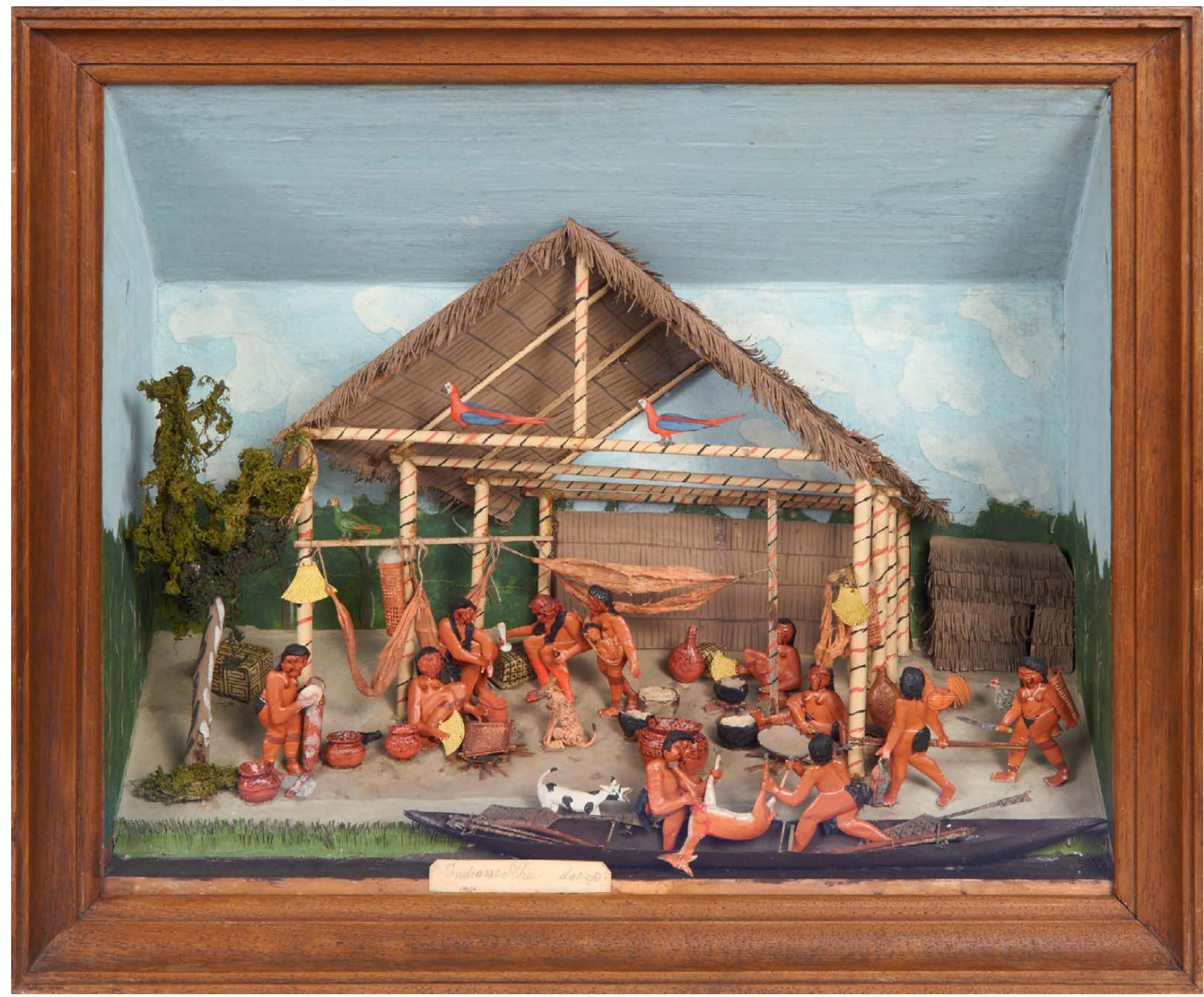

Fig. 7. Vue générale du diorama de Dunkerque après intervention. @ C2RMF/A. Chauvet. 


\section{Notes}

1. République d'Amérique du Sud jouxtant la Guyane. Durant une longue période, elle a été une colonie néerlandaise.

2. Campagne d'examens et d'imagerie scientifique accompagnée d'analyses non destructives in situ et sur prélèvements.

3. Ces représentations miniaturisées ou non, avec éléments en trois dimensions devant un fond créant l'illusion du milieu naturel, ont beaucoup été utilisées dans les musées durant la première moitié du $\mathrm{xx}^{\mathrm{e}}$ siècle.

4. D'après l'experte néerlandaise Clazien Medendorp, le diorama de Dunkerque ne serait pas de G. Schouten, alors qu'il lui a longtemps été attribué. Les proportions des personnages, la décoration des poteaux, ainsi que la réalisation du sol et la mise en peinture des côtés sont autant d'éléments qui diffèrent des réalisations de Schouten. On retrouve ces caractéristiques stylistiques sur le diorama de Cayenne.

5. Les Bray font partie de ces familles de donateurs dunkerquois - négociantsarmateurs - qui remirent au musée nombre d'objets entre 1850 et 1884, dont deux autres Carbets indiens, non attribués.

6. Registre d'entrée du musée communal de Dunkerque, antérieur à 1873 (non daté) et étiquette apposée sur l'objet. Dorp signifie village en néerlandais.

7. L'identification a été réalisée en 2007 par l'archéologue Stéphen Rostain.

8. L'identification a été permise grâce à Damien Davy, spécialiste de la vannerie amérindienne dans les Guyanes et l'anthropologue Gérard Collomb, lors des divers échanges au moment de la restauration du diorama.

9. Cousin d'Alphonse Bray et installé en 1841 au Suriname comme " officier blanc » puis planteur avant de devenir un administrateur dans la colonie néerlandaise de Guyane. Il fit également partie d'un groupe d'artistes connus. Quelques-unes de ses planches aquarellées répertoriées dans des collections publiques et privées en France et à l'étranger illustrent avec naturalisme des scènes de marronnage.

10. Pressage de la pulpe dans une grande vannerie en forme de tube, pour en extraire le jus toxique, tamisage dans une vannerie plate, puis cuisson sur une plaque métallique.

11. L'analyse plus poussée de cette terre n'a pas été entreprise car la quantité importante de matière nécessaire à l'analyse et l'absence d'échantillon de référence pour la zone géographique concernée n'auraient pas permis d'obtenir un résultat significatif.
12. L'abiétane est un diterpène majoritaire dans les résines de la famille des Pinaceae sp., voir Mills, White, 1994.

13. Anderson, Karmalla, 1966 ; Bleton et al., 1996 ; Méjanelle, 1996.

14. Cette hypothèse n'a pu être vérifiée du fait du manque d'accessibilité pour prélever.

15. Claude Steen-Guélen, responsable de collections et de la conservationrestauration - musée des Beaux-Arts et Laac, Dunkerque, et Régis Prévot, ingénieur d'étude, C2RMF.

16. Ces échanges ont aussi permis de lever rapidement les doutes engendrés par l'aspect repeint récent des jambes de cette femme. Cet effet coloré correspond aux bandelettes de tresse qui ceignent les mollets des femmes dans la tradition Kali'na.

\section{Bibliographie}

Anderson D. M. W., Karamalla K. A., 1966 "Studies on uronic acid materials", Par XII, The composition of Acacia Gum Exudates, Journal of the Chemical Society, Section C., p. 762

Bleton J., Méjanelle P., Sansoulet J., Tchapla A., 1996, "Characterization of neutral sugars and uronic acids after methanolysis and trimethylsilylation for recognition of plant gums", Journal of Chromatography A, 720: 27-49.

Bray T., 1911, « L'Émancipation des Noirs dans la Guyane hollandaise - extrait du journal d'un planteur dunkerquois, Théodore Bray, établi à Surinam en 1863 ", Société historique et archéologique de Dunkerque et de la Flandre maritime 2 (3), p. 239-259.

Caredda B. (dir.), 1990, La Grande Encyclopédie de la Caraïbe (8), p. 207.

Collomb G., 2006, Les Indiens de la Sinnamary. Journal du père Jean de la Mousse en Guyane (1684-1691), éditions Chandeigne, Paris.

Collomb G., 2015, « Les Peuples qui habitent ces rivières... ", Archéologie de l'Amazonie. Les premiers habitants de la Guyane côtière, Rostain S. (dir.), BAR International Series 2758, p. 167-173.

Collomb G., Tiouka F., 2000, Na'na kali'na Une histoire des Amérindiens Kali'na en Guyane, Ibis Rouge Éditions.

Collomb G., Van den Bel M., 2014, Entre deux mondes. Amérindiens et Européens sur les côtes de Guyane, avant la colonie (15601627), CTHS, Paris.
Medendorp C., 1999, Gerrit Schouten (17791839), Botanische tekeningen en diorama's uit Suriname, Koninklijk Instituut voor de Tropen, Amsterdam.

Medendorp C., 2008, Kijkkasten uit Suriname: de diorama's van Gerrit Schouten, Koninklijk Instituut voor de Tropen, Amsterdam.

Mills J. S., White R., 1994, The Organic Chemistry of Museum Objects, Butterworth-Heinemann, Oxford

Rostain S. (dir.), 2015, Archéologie de l'Amazonie. Les premiers habitants de la Guyane côtière, BAR International Series 2758, Paris.

Rostain S., 2016, «Amazonie, une terre promise pour l'archéologie », Pour la science, 463, Paris, p. 62-69.

Rostain S., 2016, Amazonie. Un jardin sauvage ou une forêt domestiquée. Essai d'écologie historique, Actes Sud/Errance, Arles.

Rostain S., 2017, Amazonie. Les 12 travaux des civilisations précolombiennes, coll. Science à plume, Belin, Paris

Steen-Guélen C., 2008, « Le musée des Beaux-Arts de Dunkerque : parcours de ses collections non-européennes ", Musées Ẽ collections publiques de France 2 (254), p. 49-54.

Tomasek M. (dir.), 2013, Dictionnaire biographique dunkerquois, Société Dunkerquoise d'Histoire et d'Archéologie/Les Corsaires Dunkerquois, p. 153-154.

Van Renselaar H. C., 1968, "Théodore Bray, planter and draughtsman in Surinam", Tropical Man 1, p. 140-151.

\section{Documents inédits}

Beaufils A., 2011, Les dioramas à travers l'exemple du musée des Cultures guyanaises de Cayenne : conservation-restauration, mémoire d'étude, École du Louvre.

Cadot L., Walle S., 2016, ConservationRestauration d'un diorama d'origine surinamo-néerlandaise - Inv. BA 1972.00.304, rapport d'intervention, musée des Beaux-Arts de Dunkerque.

Méjanelle P., 1996, Contribution à l'étude de substances organiques (gommes, résines et corps gras) mises en ouvre pour la réalisation d'objets d'art et d'archéologie : caractérisation par couplage chromatographie en phase gazeusespectrométrie de masse, thèse de doctorat, université Paris VI.

Vandenberghe Y., Langlois J., 2012, Étude en cours de restauration du diorama (inv. BA 1972-00-304), rapport d'analyse C2RMF $\mathrm{n}^{\circ} 25307$. 